\title{
A New Algorithm for System of Integral Equations
}

\author{
Abdujabar Rasulov, ${ }^{1}$ Adem Kilicman, ${ }^{2}$ Zainidin Eshkuvatov, ${ }^{2}$ and Gulnora Raimova ${ }^{1}$ \\ ${ }^{1}$ Department of Mathematical Modeling and Informatics, University of World Economy and Diplomacy, 100077 Tashkent, Uzbekistan \\ ${ }^{2}$ Department of Mathematics and Institute for Mathematical Research, Universiti Putra Malaysia (UPM), \\ 43400 Serdang, Selangor, Malaysia
}

Correspondence should be addressed to Adem Kilicman; akilic@upm.edu.my

Received 30 April 2014; Revised 17 June 2014; Accepted 18 June 2014; Published 14 August 2014

Academic Editor: Hari M. Srivastava

Copyright (C) 2014 Abdujabar Rasulov et al. This is an open access article distributed under the Creative Commons Attribution License, which permits unrestricted use, distribution, and reproduction in any medium, provided the original work is properly cited.

We develop a new algorithm to solve the system of integral equations. In this new method no need to use matrix weights. Beacause of it, we reduce computational complexity considerable. Using the new algorithm it is also possible to solve an initial boundary value problem for system of parabolic equations. To verify the efficiency, the results of computational experiments are given.

\section{Introduction}

The theory and application of integral equations are an important subject within pure and applied mathematics and they appear in various types in many fields of science and engineering. The integral equations can also be represented as convolution integral equations; see Srivastava and Buschman [1]. In the applications, the number of computational problems can be reduced to the solution of a system of integral equations (system of IEs) of the second kind; see [2-4]. However, solving systems of integrodifferential equations are very important and such systems might be difficult analytically, so many researchers have attempted to propose different numerical methods which are accurate and efficient. For example, numerical expansion methods for solving a system of linear IDEs by interpolation and Clenshaw Curtis quadrature rules were presented in [5], where the integral system was transferred into a matrix equation by the interpolation points. Pour in [6] studied an extension of the Tau method to obtain the numerical solution of Fredholm integrodifferential equations systems ad applied Chebyshev basis to solve IDEs. Similarly, Arikoglu and Ozkol [7] obtained solutions of integral and integrodifferential equation systems by using differential transform method where the approch provides very good approximation to the exact solution.

Recently, the solution of the system has been estimated by many different basic functions, such as orthonormal bases and wavelets; see, for example [8,9], and the hybrid Legendre Block-Pulse functions, that is, a combination of the Block-Pulse functions on $[0,1]$ and Legendre polynomials was proposed. In addition, the Bessel matrix method was introduced in [10] for solving a system of high order linear Fredholm differential equations with variable coefficients. In the literature there are several methods to solve the different type of integral equations; see [11-16]. One of the novel methods is known as the vector Monte Carlo algorithms to solve the system of IEs. Among the vector Monte Carlo algorithms the following are well known:

(i) an algorithm for solving the system of transfer equations with polarization;

(ii) a vector algorithm for solving multigroup transfer equations;

(iii) a Monte Carlo technique combined with the finite sum method and vector Monte Carlo method for solving metaharmonic equations.

In the use of this method one can easily see that the variance of the vector estimate largely depends on the form of transitional density. Thus appropriate choice of the density leads to the reduction of the complexity calculations, which is defined as the product of the variance and the computational time. To determine the density is difficult as to solve the problem itself, although in some cases it is possible to obtain 
a minimal criterian of uniform optimality of the method. The transitional density that corresponds to minimum complexity of algoritm is said to be optimal for a given problem.

In Mikhailov [17], vector Monte Carlo algorithms are used to solve system of IEs. The distinguished feature of that vector algorithm is that its "weight" appears in the form of a matrix weight. This matrix weight is multiplied by the kernel matrix of the system of IEs dividing by a transition density function in the Markov chain simulation, so that a number of computational problems can be reduced to the solution of a system of IEs of second kind. By introducing a suitable discrete-continuous measure of the integration, we can write the system of IEs in the form of a single integral equation, and this allows us to use standard algorithms of the Monte Carlo method. However, it is more expedient to make use of the matrix structure of the system and solve the problem by the Monte Carlo method with vector weights. The following vector Monte Carlo algorithms are well known: an algorithm for solving the system of transfer equations with polarization taken into account, a vector algorithm for solving multigroup transfer equations, a Monte Carlo technique combined with the finite sum method, and vector Monte Carlo method for solving metaharmonic equation.

In this study, a new algorithm is proposed for the numerical solution of system of IEs but in this algorithm we do not use matrix weights. The proposed algorithm has usual advantages of ordinary Monte Carlo method. The new algorithm is considerably reduced to computational complexity. Using this new algorithm we have solved an initial boundary value problem for system of parabolic equations. The paper is organized as follows. In Section 2, we present the description of the problem and proposed a new Monte Carlo algorithm for the solution of system of IEs. In Section 3, we discuss the application of the method to the solution of system of parabolic equations. In Section 4, we will construct biased and $\varepsilon$-biased estimators for the solution. In Section 5, the results of computational experiments are given, followed by the conclusion in Section 6.

\section{Description of the Problem}

\section{and a New Approach for the Solution of System of IEs}

Let us consider second kind nonhomogeneous system of IEs of the form

$$
\varphi_{i}(x)=\sum_{j=1}^{n} \int_{X} k_{i, j}(x, y) \varphi_{j}(y) d y+h_{i}(x), \quad i=1, \ldots, n,
$$

where $x \in X \subseteq \mathbb{R}^{m}, m \geq 1$ or in vector form

$$
\Phi=K \Phi+H
$$

here operator $K: L_{\infty} \rightarrow L_{\infty}$ where $L_{\infty}$ is the space of bounded function almost everywhere and

$$
\begin{gathered}
H=\left(h_{1}, \ldots, h_{n}\right) \in L_{\infty}, \quad K=\left(K_{i j}\right) \in L_{\infty}, \\
\Phi=\left(\varphi_{1}, \varphi_{2}, \ldots, \varphi_{n}\right) \in L_{\infty},
\end{gathered}
$$

where the norm of $H$ is

$$
\|H\|_{L_{\infty}}=\operatorname{vrai}_{1 \leq i \leq n, x \in X}\left|h_{i}(x)\right| .
$$

Suppose the spectral radius $\rho(K)$ satisfy the inequalities

$$
\rho(K)=\lim _{n \rightarrow \infty}\left\|K^{n}\right\|^{1 / n}<1, \quad \rho(K) \leq\left\|K^{n}\right\|^{1 / 2},
$$

where $K^{n} \varphi=K^{n-1} K \varphi$.

Let Markov chain $\left\{x_{n}, n=0,1, \ldots, N\right\}$ with transition density $p(x, y)$ be

$$
g(x)=1-\int_{X} p(x, y) d y \geq 0,
$$

where $g(x)$ is the probability of absorption at the point $x_{N}$, where $N$ is the random number of the last moment and in initial moment $x_{0}=x$.

A standard vector algorithm of Monte Carlo for $\Phi(x)$ is

$$
\begin{aligned}
& \Phi(x)=\left(M \xi_{x}, \xi_{x}\right)=H(x)+\sum_{n=1}^{N} Q_{n} H\left(x_{n}\right), \\
& Q_{0}=I, \quad Q_{n+1}=\frac{Q_{n} K\left(x_{n}, x_{n+1}\right)}{P\left(x_{n}, x_{n+1}\right)}, \quad n=0,1,2, \ldots,
\end{aligned}
$$

where $I$ is a unit matrix, $K(x, y)$ is a kernel matrix $\left\{k_{i j}(x, y)\right\}$, and $p\left(x_{n}, x_{n+1}\right)$ is the transition density function at the points $\left(x_{n}, x_{n+1}\right)$. The condition for unbiasedness is

$$
p\left(K_{1}\right)<1 \quad \text { or } K_{1}=\|K\|<1 .
$$

We will assume also that the spectral radius of the operator $K_{1}$ obtained from $K$ by the substitution $k_{i, j} \rightarrow\left|k_{i, j}\right|$ is less than one. Then, by using standard methods of Monte Carlo theory we can show that

$$
\begin{aligned}
& \Phi(x)=E \xi_{x}, \quad \xi_{x}=\sum_{n=0}^{N} Q_{n} H\left(x_{n}\right) \\
& Q_{0}=\left\{\delta_{i, j}\right\}_{i, j=(1, \ldots, n)}, \quad Q_{n}=Q_{n-1} \frac{K\left(x_{n-1}, x_{n}\right)}{p\left(x_{n-1}, x_{n}\right)},
\end{aligned}
$$

where $Q_{n}$ can be considered as matrix weight and

$$
\frac{K\left(x_{n-1}, x_{n}\right)}{p\left(x_{n-1}, x_{n}\right)}=\left\{\frac{k_{i j}\left(x_{n-1}, x_{n}\right)}{p\left(x_{n-1}, x_{n}\right)}\right\}, \quad i, j=\{1,2, \ldots, n\} .
$$

The Monte Carlo method is used to estimate linear functionals of the form

$$
(F, \Phi)=\int_{X} F^{\prime}(x) \Phi(x) d x
$$

where $F^{\prime}(x)=\left(f_{1}(x), f_{2}(x), \ldots f_{n}(x)\right)$ with

$$
\|F\|_{L_{1}}=\sum_{j=1}^{n} \int_{X}\left|f_{j}(x)\right| d x<\infty
$$


Let the point $x_{0}$ be distributed with initial probability density $\pi(x)$ such that

$$
\pi(x) \neq 0 \quad \text { if } F^{\prime}(x) \Phi(x) \neq 0
$$

Then, obviously (see Mikhailov [17]),

$$
\begin{aligned}
(F, \Phi) & =E\left[\frac{F^{\prime}\left(x_{0}\right)}{\pi\left(x_{0}\right)} \xi_{x_{0}}\right]=E\left[\sum_{n=0}^{N} \frac{F^{\prime}\left(x_{0}\right)}{\pi\left(x_{0}\right)} Q_{n} H\left(x_{n}\right)\right] \\
& =E\left[\sum_{n=0}^{N} H^{\prime}\left(x_{n}\right) Q_{n}^{\prime} \frac{F^{\prime}\left(x_{0}\right)}{\pi\left(x_{0}\right)}\right] .
\end{aligned}
$$

The random vector with weight $Q_{n}^{(1)}=Q_{n}^{\prime}\left(F^{\prime}\left(x_{0}\right) / \pi\left(x_{0}\right)\right)$ is computed by the formula

$$
Q_{n}^{(1)}=\frac{K^{\prime}\left(x_{n-1}, x_{n}\right)}{p\left(x_{n-1}, x_{n}\right)} Q_{n-1}^{(1)}
$$

Precisely such a vector algorithm, corresponding to the representation $I=\left(\Phi^{*}, H\right)$, has been formulated in the work of Mikhailov [17]. Below on the contrary to vector algorithms we will propose a new algorithm for the solution of system of integral equations. Our method does not use matrix weights.

Suppose we have to find the solution of the inhomogeneous system of IEs (1) of the second kind at the point $x \in X$. We will define two types of Markov chain $\left\{i_{K}\right\}$ and $\left\{x_{K}\right\}$ by the following way.

(a) Definition of the First Homogeneous Markov Chain. Now we simulate the Markov chain $i_{0}, i_{1} \cdots \in N$ with $n+1$ state. Initial state $i_{0}$ will simulate according to initial distribution $\pi=\left(\pi_{1}, \ldots, \pi_{n}, 0\right)$ and the next $i_{1}$ with the transition matrix

$$
\begin{gathered}
A=A(x)=\left\|\alpha_{i, j}(x)\right\|_{i, j=1}^{n+1}, \\
\sum_{j=1}^{n} \alpha_{i, j}(x)=1-g_{i}(x), \\
g_{i}(x)=\alpha_{i, n+1}(x), \quad i=1, \ldots, n .
\end{gathered}
$$

Here $\alpha_{n+1, n+1}(x)=1$ and

$$
A(x)=\left(\begin{array}{cc}
\alpha_{11}(x), \ldots, \alpha_{1 n}(x), & g_{1}(x) \\
\alpha_{21}(x), \ldots, \alpha_{2 n}(x), & g_{2}(x) \\
\alpha_{n 1}(x), \ldots, \alpha_{n n}(x), & g_{n}(x) \\
0, \ldots, 0, & 1
\end{array}\right)
$$

Let $N$ be a random absorption moment with $N=$ $\left\{\max k, i_{k} \neq n+1\right\}$, a life time of chain.

(b) A second homogeneous Markov chain $\left\{x_{k}\right\}$ with space phase $X$ is defined by the following way.
Firstly, we define the transition density matrix as

$$
\begin{gathered}
P\left(x^{1} \longrightarrow x\right)=\left\|P_{i, j}\left(x^{1} \longrightarrow x\right)\right\|_{i, j=1}^{n+1}, \\
P\left(x^{1} \longrightarrow x\right)=\left(\begin{array}{cc}
P_{11}\left(x^{1} \longrightarrow x\right), \ldots, P_{1 n}\left(x^{1} \longrightarrow x\right), & 0 \\
P_{21}\left(x^{1} \longrightarrow x\right), \ldots, P_{2 n}\left(x^{1} \longrightarrow x\right), & 0 \\
P_{n 1}\left(x^{1} \longrightarrow x\right), \ldots, P_{n n}\left(x^{1} \longrightarrow x\right), & 0 \\
0, \ldots, 0, & 1
\end{array}\right) .
\end{gathered}
$$

Let an initial point $x_{0}=x$; using $\pi(x)$ we will simulate initial moment $i_{0}$, then according to the transition matrix $A\left(x_{0}\right)$ we are able to simulate again the next state of chain $i_{1}$. It means with the probability $\alpha_{i_{0}, i_{1}}\left(x_{0}\right), P\left(i_{1}=k\right)=\alpha_{0 k}\left(x_{0}\right)$.

The next phase coordinates of the chain $x_{1}$ simulated according to $p_{i_{0}, i_{1}}\left(x_{0}, x_{1}\right)$. The probability of absorption of the trajectory is $g_{i_{0}}\left(x_{0}\right)$. Let $\left(i_{k}, x_{k}\right)$ be known then the next value of $i_{k+1}$ will be defined according to the matrix $A\left(x_{k}\right)$ and next random point $x_{k+1}$ simulated according to the probability density function $P_{i_{k}, i_{k+1}}\left(x_{k}^{1} \rightarrow x\right)$ and so on.

Let $\xi_{x_{0}}=\xi_{N}\left(i_{0}, i_{1}, \ldots i_{N} ; x_{0}, x_{1}, \ldots x_{N}\right)$ be some random variable which is defined by the set of trajectory Markov chains. The mathematical expectations of random variable will be

$$
\begin{gathered}
E \xi_{x_{0}}=\sum_{k=0}^{\infty} \sum_{i_{0}, . . i_{k}=1}^{n} \underbrace{\int \cdots \int}_{k} \pi_{i_{0}} \alpha_{i_{0}, i_{1}}\left(x_{0}\right) p_{i_{0}, i_{1}}\left(x_{0} x_{1}\right) \cdot \alpha_{i_{1}, i_{2}}\left(x_{1}\right) \\
p_{i_{1}, i_{2}}\left(x_{1}, x_{2}\right) \cdots \alpha_{i_{k-1}, i_{k}}\left(x_{k-1}\right) \\
\quad \times p_{i_{k-1}-1, i_{k}}\left(x_{k-1}, x_{k}\right) g_{i_{k}}\left(x_{k}\right) \\
\xi_{k}\left(i_{0}, \ldots i_{k}, x_{0}, \ldots x_{k}\right) d x_{1} \cdots d x_{k} .
\end{gathered}
$$

Let us consider calculation of the functional $(\Phi, F)$, where $F^{T}=\left(f_{1}(x), \ldots, f_{n}(x)\right)$ column vector. Let us compute the functional $(\Phi, F)=\sum_{i=1}^{n} \varphi_{i}(x) f_{i}(x)$. For doing this task we introduce two well-known estimators according to the Monte Carlo theory. First of them is analog of absorption estimator

$$
\begin{aligned}
\xi_{1}\left(x_{0}\right)= & \frac{f_{i_{0}}\left(x_{0}\right)}{\pi_{i_{0}}\left(x_{0}\right)} \frac{k_{i_{0} i_{1}}\left(x_{0}, x_{1}\right)}{\alpha_{i_{0}, i_{1}}\left(x_{0}\right) p_{i_{0}, i_{1}}\left(x_{0}, x_{1}\right)} \\
& \cdots \frac{k_{i_{n-1}, i_{n}}\left(x_{n-1}, x_{n}\right)}{\alpha_{i_{n-1}, i_{n}}\left(x_{n-1}\right) p_{i_{n-1}, i_{n}}\left(x_{n-1}, x_{n}\right)} \cdot \frac{h_{i_{n}}\left(x_{n}\right)}{g_{i_{n}}\left(x_{n}\right)}
\end{aligned}
$$

and the second one is analog of collision estimator

$$
\begin{aligned}
\xi_{2}\left(x_{0}\right)= & \sum_{j=1}^{N} \frac{f_{i_{0}}\left(x_{0}\right)}{\pi_{i_{0}}\left(x_{0}\right)} \frac{K_{i_{0} i_{1}}\left(x_{0}, x_{1}\right)}{\alpha_{i_{0}, i_{1}}\left(x_{0}\right) p_{i_{0}, i_{1}}\left(x_{0}, x_{1}\right)} \\
& \cdots \frac{K_{i_{j-1}, i_{j}}\left(x_{j-1}, x_{j}\right)}{\alpha_{i_{j-1}, i_{j}}\left(x_{j-1}\right) p_{i_{j-1}, i_{j}}\left(x_{j-1}, x_{j}\right)} \cdot h_{i_{j}}\left(x_{j}\right) .
\end{aligned}
$$


Theorem 1. If $f_{i_{0}}\left(x_{i_{0}}\right) \neq 0$ then $\pi_{i_{0}}\left(x_{i_{0}}\right) \neq 0$ and if $k_{i_{i} i_{j}}\left(x_{i}, x_{j}\right) \neq 0$ then

$$
\alpha_{i, j}\left(x_{i}\right) p_{i_{i} i_{j}}\left(x_{i}, x_{j}\right) \neq 0, \quad \text { for any } 1 \leq i, j \leq n .
$$

In this case $E \xi_{1,2}=(\Phi, F)$.

The proof of the theorem is similar to the theorem Ermakov [23], and therefore proof is omitted. Now we will apply the obtained results to the solution system of parabolic equations.

\section{Application to System of Parabolic Equations}

In this section we consider initial boundary problem for system of parabolic equations. Let $D$ be bounded domain in $R^{m}$ with enough smooth boundary $\partial D$ of $\Omega=D \times[0, T]$ and $\Omega$ is the cylinder in $R^{m+1}$ with parallel spin axis $t$. The basement is the domain $D$ on the surface $t=0$ and $T$ is the fixed constant. The functions

$$
\begin{gathered}
y_{0 i}(x) \in C(\bar{D}), \quad y_{i}(x, t) \in C(\partial D \times[0, T]), \\
f_{i}(x, t) \in C(\bar{\Omega}),
\end{gathered}
$$

where $C(\bar{D})$ stands for a continuous function on the closed domain $D$.

Now consider the following initial boundary value problem (BVP) for system of parabolic equations:

$$
\begin{gathered}
\frac{\partial u_{1}(x, t)}{\partial t}-a_{1} \Delta u_{1}(x, t)+c_{11} u_{1}(x, t) \\
-c_{12} u_{2}(x, t)-\cdots-c_{1 n} u_{n}(x, t)=f_{1}(x, t) \\
\frac{\partial u_{2}(x, t)}{\partial t}-a_{2} \Delta u_{2}(x, t)+c_{22} u_{2}(x, t) \\
-c_{21} u_{1}(x, t)-\cdots-c_{2 n} u_{n}(x, t)=f_{2}(x, t) \\
\quad \vdots \\
\frac{\partial u_{n}(x, t)}{\partial t}-a_{n} \Delta u_{n}(x, t)+c_{n n} u_{n}(x, t)-c_{n 1} u_{1}(x, t) \\
-\cdots-c_{(n-1) n} u_{n-1}(x, t)=f_{n}(x, t),
\end{gathered}
$$

where the coefficients $a_{i}>0, \quad c_{i j}>0,(i=1, \ldots, n, j=$ $1, \ldots, n)$, and $(x, t) \in \Omega$ with initial and boundary conditions

$$
\begin{aligned}
& u_{i}(x, t)=y_{i}(x, t), \quad x \in \partial D, t \in[0, T], i=\overline{1, n} \\
& u_{i}(x, 0)=y_{o i}(x), \quad x \in D, i=\overline{1, n} .
\end{aligned}
$$

Further suppose $f_{i}(x, t), y_{o i}(x), y_{i}(x, t)$, and coefficients $a_{i}, c_{i j}(i, j=\overline{1, n})$ are given such that there exists unique solution Ladyzhenskaya et al. [18] and Lions [19] of the initial BVR (24)-(25) and

$$
\begin{aligned}
& u_{i}(x, t) \in C(\bar{D} \times[0, T]) \\
& \cap C^{2,1}(\bar{D} \times[0, T]) \quad(i=\overline{1, n}),
\end{aligned}
$$

where $C^{2,1}$ is the set of continuous functions in the given region with continuous derivatives $u_{x}, u_{x x}$, and $u_{t}$.

Now we construct unbiased estimator for the problem (24)-(25) in the arbitrary point $(x, t) \in \Omega$ on the trajectory some random process. For that we use mean value formula and construct some special system of integral equations for $u_{i}(x, t)$ in special constructed domains (spheroid or balloid with the center $(x, t))$.

According to Section 2 below we will propose a new nonstationary Markov chain on which trajectory will construct unbiased estimators for the obtained system of integral equations.

In our algorithm we do not used matrix weight; it means the computational complexity of new algorithm is much better. The basis for the constructing of algorithms will be the formula of parabolic mean for the heat conductivity equations. As we know the fundamental solution $Z(x, t, y, \tau)$ for heat equation $u_{t}-a \Delta u=0$ is given by

$$
\begin{aligned}
& Z(x, t ; y, \tau) \\
& \quad=(4 \pi a(t-\tau))^{-m / 2} \exp \left(-\frac{|x-y|^{2}}{4 a(t-\tau)}\right) .
\end{aligned}
$$

Firstly, we define a special domain using a fundamental solution of the heat equation $Q_{r}(x, t)$ which depends on $r>0$ and points $(x, t) \in R^{m+1}$ as

$$
Q_{r}(x, t)=\left\{(y, \tau): Z(x, t ; y, \tau)>(4 \pi a r)^{-m / 2}, \tau<t\right\} .
$$

The domain $Q_{r}(x, t)$, we call balloid and $\partial Q_{r}(x, t)$, spheroid with the center in the point $(x, t)$. From the definition balloid $Q_{r}(x, t)$, described by following inequality (Kupcov [20]):

$$
Q_{r}(x, t)=\left\{(y, \tau):|x-y|^{2}<2 m a(t-\tau) \ln \frac{r}{t-r}, \tau<t\right\} .
$$

Each section with the sectional plain of balloid when $\tau=$ constant will be $m$-dimensional ball $B(x, R(t-\tau))$ with the center $x$ and with the radius

$$
R(t-\tau)=\sqrt{2 m a(t-\tau) \ln \frac{r}{t-r}} .
$$

Let $(x, t) \in \Omega$ and

$$
r=r(x, t)=\min \left\{\frac{R_{1}^{2}(x) e}{2 a m}, t\right\},
$$

where $R_{1}(x)$ is the minimum distance from point $x$ until the boundary; that is,

$$
R_{1}(x)=\inf \left\{\left|x-x^{\prime}\right|, x \in \partial D, x^{\prime} \in \bar{D}\right\} .
$$

In this case $Q_{r}(x, t) \subset \bar{\Omega}$. By further using Greens function and fundamental solution we will transfer from the system of differential equations into the system of integral equations. In the book [21] special balance equation analogies were constructed as in [22], which connected the value of function $u(x, t)$ with its integral from the spheroid and balloid with the center in the point $(x, t)$. 
Lemma 2 (Kurbanmuradov [22]). Let the function $u(x, t)$ satisfy the following equation:

$$
\frac{\partial u(x, t)}{\partial t}-a \Delta u(x, t)=f(x, t), \quad(x, t) \in \Omega .
$$

Then the following formula of mean is true(mean value formula):

$$
\begin{aligned}
u(x, t)=a \iint_{\partial Q_{r}(x, t)}\left(1-\frac{t-\tau}{r}\right) & \\
\quad & \times\left(-\frac{\partial Z(x, t ; y, \tau)}{\partial n_{y}}\right) u(y, \tau) d s d \tau \\
& +\frac{1}{r} \iint_{Q_{r}(x, t)} Z_{r}(x, t ; y, \tau) u(y, \tau) d y d \tau+F_{r}(x, t),
\end{aligned}
$$

where

$$
\begin{gathered}
F_{r}(x, t)=\frac{1}{r} \iint_{Q_{r}(x, t)}(r-(t-\tau)) Z_{r}(x, t ; y, \tau) f(y, \tau) d y d \tau, \\
Z_{r}(x, t ; y, \tau)=Z(x, t ; y, \tau)-(4 \pi a r)^{-m / 2},
\end{gathered}
$$

here $d s$ is the element of small area of sphere $\partial B(x, R(t-$ $\tau)$ ). In further using these results we will get special integral representation.

3.1. Transforming a System and Obtaining Integral Representation. Let us define the family of domains $Q_{r}^{(i)}(x, t)$, which depends on positive parameters $r>0$ and point $(x, t) \in R^{m+1}$, where

$$
Q_{r}^{(i)}(x, t)=\left\{(y, \tau): Z_{r}^{i}(x, t ; y, \tau)>0, \tau<t\right\},
$$

where $Z_{r}^{i}(x, t ; y, \tau)$ defined analogous $Z_{r}(x, t ; y, \tau)$ changing a for $a_{i}$ (see above Lemma 2). The domain $Q_{r}^{i}(x, t)$ we will call a balloid with radius $r$ which a center in a point $(x, t)$ and a boundary $\partial Q_{r}^{(i)}(x, t)=\left\{(y, \tau): Z_{r}^{i}(x, t ; y, \tau)=0, \tau \leq t\right\}$ is spheroid. Here

$$
r=r(x, t)=\min \left\{\frac{R_{1}^{2}(x) e}{2 a_{1} m}, \ldots, \frac{R_{1}^{2}(x) e}{2 a_{n} m}, t\right\} .
$$

Let $(x, t) \in \Omega$ and $D: R_{1}(x)=\inf \left\{\left|x-x^{\prime}\right|, x \in \partial D, x^{\prime} \in\right.$ $\bar{D}$ \} where $R_{1}(x)$ is the distance from the point $(x, t)$ to the boundary of domain. In this case $Q_{r}^{i}(x, t) \subset \bar{\Omega}$. Appling the expression (34) to each of the equations we will get the following system of integral equations $(i=1,2, \ldots, n)$ :

$$
\begin{aligned}
& u_{i}(x, t) \\
& =a_{i} \iint_{\partial Q_{r}^{i}(x, t)}\left(1-\frac{t-\tau}{r}\right)\left(\frac{\partial Z^{(i)}(x, t ; y, \tau)}{\partial n_{y}}\right) u_{i}(y, \tau) d s d \tau
\end{aligned}
$$

$$
\begin{aligned}
& +\frac{1}{r} \iint_{\partial Q_{r}^{i}(x, t)}\left(1-\left(r-(t-\tau) c_{i i}\right)\right) \\
& \times Z_{r}^{(i)}(x, t ; y, \tau) u_{i}(y, \tau) d y d \tau \\
& +\frac{1}{r} \iint_{\partial Q_{r}^{i}(x, t)}(r-(t-\tau)) Z_{r}^{(i)}(x, t ; y, \tau) \\
& \times \sum_{j=1, n ; i \neq j} c_{i j} u_{j}(y, \tau) d y d \tau \\
& +\frac{1}{r} \iint_{Q_{r}^{i}(x, t)}(r-(t-\tau)) Z_{r}^{(i)}(x, t ; y, \tau) f_{i}(y, \tau) d y d \tau, \\
& \quad i=1, \ldots, n,
\end{aligned}
$$

where

$$
\begin{gathered}
Z^{(i)}(x, t ; y, \tau)=\left(4 \pi a_{i}(t-\tau)\right)^{-m / 2} \exp \left(-\frac{|x-y|^{2}}{4 a_{i}(t-\tau)}\right), \\
Z_{r}^{i}(x, t ; y, \tau)=Z^{(i)}(x, t ; y, \tau)-\left(4 \pi a_{i}(t-\tau)\right)^{-m / 2} .
\end{gathered}
$$

The derived system (38) is similar to system IEs which was considered in Section 2. That is way we can use the method which was given in Section 2.

3.2. The Probabilistic Representation of the Solution. After some transformation we will get for separate terms of the system (38) as follows:

$I_{1}^{(i)}(x, t)$

$$
\begin{aligned}
& =a_{i} \iint_{\partial Q_{r}^{i}(x, t)}\left(1-\frac{t-\tau}{r}\right)\left(-\frac{\partial Z^{(i)}(x, t ; y, \tau)}{\partial n_{y}}\right) u_{i}(y, \tau) d s d \tau \\
& =\left(1-q_{m}\right) \int_{0}^{\infty} q_{1}(\rho) d \rho \int_{S_{1}(0)} q_{2}(\omega) u_{i}\left(y^{(i)}(\rho, \omega), \tau(\rho)\right) d s \\
& =\left(1-q_{m}\right) E u_{i}\left(y^{(i)}(\xi, \omega), \tau(\xi)\right),
\end{aligned}
$$

where

$$
\begin{aligned}
& q_{1}(\rho)= \rho^{m / 2} \exp (-\rho)\left(1-\exp \left(-\frac{2 \rho}{m}\right)\right) \\
& \times\left(\left(1-q_{m}\right) \Gamma\left(1+\frac{m}{2}\right)\right)^{-1}, \\
& q_{2}(\omega)= \frac{1}{\sigma_{m}}=\Gamma\left(\frac{m}{2}\right)\left(2 \pi^{m / 2}\right)^{-1}, \\
& q_{m}=\left(1+\frac{2}{m}\right)^{-(1+m / 2)}
\end{aligned}
$$




$$
\begin{aligned}
& y^{(i)}(\xi, \omega)=x+\sqrt{4 r \xi a_{i} \exp \left(-\frac{2 \xi}{m}\right) \omega,} \\
& \tau(\xi)=t-r \exp \left(-\frac{2 \xi}{m}\right),
\end{aligned}
$$

$\xi$ is a random variable with density functions $q_{1}(\rho), \omega$ random point on the surface $S_{1}(0)$, which has a density function $q_{2}(w), S_{1}(0)$ unit sphere, ds element of surface, $\sigma_{m}$ square of the surface unit sphere, and $\Gamma(\cdot)$ Gamma function.

Let us consider the second terms of (38)

$$
\begin{aligned}
I_{2}^{(i)}(x, t)= & \frac{1}{r} \iint_{Q_{r}^{i}(x, t)}(r-(t-\tau)) Z_{r}^{i}(x, t ; y, \tau) f_{i}(y, \tau) d y d \tau \\
= & r q_{m} \int_{0}^{1} q_{3}(\nu) d \nu \int_{0}^{\infty} q_{4}(z) d z \\
& \cdot \int_{S_{1}(0)}\left(1-v^{2 / m} \exp \left(\frac{-2 z}{m+2}\right)\right) \\
\times & f_{i}\left(y_{1}^{(i)}(z, v, w), \tau_{1}(z, \nu)\right) d S_{w} \\
=r q_{m} E & \left\{\left(1-v^{2 / m} \exp \left(\frac{-2 \xi_{1}}{m+2}\right)\right)\right. \\
& \left.\times f_{i}\left(y_{1}^{(i)}\left(\xi_{1}, v, \omega\right), \tau_{1}\left(\xi_{1}, v\right)\right)\right\},
\end{aligned}
$$

where $q_{3}(\nu)=(1-\nu) v^{2 / m-1}(B(2,2 / m))^{-1}$. Then the density of Beta distribution with parameters $(2,2 / m)$

$$
q_{4}(z)=\exp (-z) z^{m / 2-1}\left(\Gamma\left(\frac{m}{2}\right)\right)^{-1},
$$

and the density of Gamma distribution with parameters $m / 2$, $\omega$-unit random vector,

$$
\begin{aligned}
& y_{1}^{i}\left(\xi_{1}, v, \omega\right)=x+\left[\frac{4 m}{m+2} r a_{i} \xi_{1} v^{2 / m} \exp \left(-\frac{2 \xi_{1}}{m+2}\right)\right]^{1 / 2} \omega \\
& \tau_{1}\left(\xi_{1}, v\right)=t-r v^{2 / m} \exp \left(-\frac{2 \xi_{1}}{m+2}\right),
\end{aligned}
$$

where $\xi_{1}$ is the random variable with density function $q_{4}(z)$ and $v$ is another random variable with the density function $q_{3}(\nu)$.

$$
r=r(x, t)=\min \left\{\frac{e R_{1}^{2}(x)}{2 m a_{1}}, \ldots, \frac{e R_{1}^{2}(x)}{2 m a_{n}} ; \frac{1}{c_{11}}, \ldots, \frac{1}{c_{n n}} ; t\right\} \text {, }
$$

then $\overline{Q_{r}^{(i)}}(x, t) \in \Omega$ and the function

$$
\begin{aligned}
p_{1}^{(i)}(x, t ; y, \tau)= & \frac{\left[1-(r-(t-\tau)) c_{i}\right] Z_{r}^{(i)}(x, t ; y, \tau)}{r q_{m}\left(1-r q_{1 m} c_{i i}\right)} \\
& \times I_{Q_{r}^{(i)}(x, t)}(y, \tau)
\end{aligned}
$$

is the transition density in $Q_{r}^{i}(x, t)$ with fixed point $(x, t)$, where

$$
q_{1 m}=1-\frac{1}{2}\left(\frac{m+2}{m+4}\right)^{1+m / 2} .
$$

Let $\left(y_{2}^{(i)}, \tau_{2}^{(i)}\right)$ be a random point of balloid $Q_{r}^{i}(x, t)$ which has the following density function $p_{1}^{(i)}(x, t ; y, \tau)(i=1, \ldots, n)$ in the fixed point $(x, t)$.

In this case

$$
\begin{aligned}
& \frac{1}{r} \iint_{Q_{r}^{(i)}(x, t)}\left(1-(r-(t-\tau)) c_{i i}\right) Z_{r}^{(i)}(x, t ; y, \tau) u_{i}(y, \tau) d y d \tau \\
& \quad=q_{m}\left(1-r c_{i i} q_{1 m}\right) E u_{i}\left(y_{2}^{(i)}, \tau_{2}^{(i)}\right), \quad i=1, \ldots, n
\end{aligned}
$$

The obtained results we will put to (38) and we will get the probabilistic representation of problem (24)-(25). It follows there from that we could to following proposition.

Theorem 3. For the solution of initial BVP (24)-(25) the following probabilistic representation is valid:

$$
\begin{aligned}
u_{i}(x, t)=\left(1-q_{m}\right) E u_{i}\left(y^{(i)}(\xi, \omega), \tau(\xi)\right) \\
+q_{m}\left(1-r c_{i i} q_{1 m}\right) E u_{i}\left(y_{2}^{(i)}, \tau_{2}^{(i)}\right) \\
+q_{m} r E\left\{\left(1-v^{2 / m} \exp \left(-\frac{2 \xi_{1}}{m+2}\right)\right)\right. \\
\left.\quad \times \sum_{j=1, j \neq i}^{n} c_{i j} u_{j}\left(y_{1}^{(i)}\left(\xi_{1}, v, \omega,\right), \tau_{1}\left(\xi_{1}, v\right)\right)\right\} \\
+q_{m} r E\left(\left(1-v^{2 / m} \exp \left(-\frac{2 \xi_{1}}{m+2}\right)\right)\right. \\
\left.\quad \times f_{i}\left(y_{1}^{(i)}\left(\xi_{1}, v, \omega\right), \tau_{1}\left(\xi_{1}, v\right)\right)\right), \quad(i=\overline{1, n}),
\end{aligned}
$$

where $\left(y^{(i)}(\xi, \omega), \tau(\xi)\right)$ is defined by (40) and $\left(y^{(i)}(\xi, v, \omega), \tau(\xi, v)\right)$ is determined by $(41)$.

The proof of Theorem 3 is the consequence of the above mentioned reasoning.

By further using the presentation (44) we will construct a random process in $\Omega$ and propose the Monte Carlo algorithm for the solution of system IEs.

3.3. Description Random Process and the Algorithm Simulation. Let

$$
r=r(x, t)=\min \left\{\frac{e R_{1}^{2}(x)}{2 m a_{1}}, \ldots, \frac{e R_{1}^{2}(x)}{2 m a_{n}} ; \frac{1}{c_{11}}, \ldots, \frac{1}{c_{n n}} ; t\right\},
$$


The functions

$$
\begin{aligned}
p_{0}^{(i)}(x, t ; y, \tau)= & \frac{1}{1-q_{m}}\left(1-\frac{t-\tau}{r}\right)\left(-\frac{\partial Z^{(i)}(x, t ; y, \tau)}{\partial n_{y}}\right) \\
& \times I_{\partial Q_{r}^{(i)}(x, t)}(y, \tau), \\
p_{1}^{(i)}(x, t ; y, \tau)= & \frac{\left(1-(r-(t-\tau)) c_{i i}\right) Z_{r}^{(i)}(x, t ; y, \tau)}{r q_{m}\left(1-r q_{1 m} c_{i i}\right)} \\
& \times I_{\partial Q_{r}^{(i)}(x, t)}(y, \tau), \\
p_{2}^{(i)}(x, t ; y, \tau)= & \frac{Z_{r}^{(i)}(x, t ; y, \tau)}{r q_{m}} I_{\partial Q_{r}^{(i)}(x, t)}(y, \tau),
\end{aligned}
$$

are the transition density functions in $\overline{Q_{r}^{(i)}}(x, t)$ at a fixed point $(x, t)(i=\overline{1, n})$. We will define in $\Omega$ a random process as was proposed in Section 2.

Let us define a transition matrix as

$$
A(x, t)=\left[\begin{array}{cccc}
\alpha_{11} & \alpha_{12} & \ldots & \alpha_{1(n+1)} \\
\alpha_{21} & \alpha_{22} & \ldots & \alpha_{2(n+1)} \\
\ldots & \ldots & \ldots & \ldots \\
\alpha_{n 1} & \alpha_{n 2} & \ldots & \alpha_{n(n+1)} \\
0 & 0 & \ldots & 1
\end{array}\right]
$$

where $\alpha_{i i}=1-q_{m} c_{i i} q_{1 m} r(x, t)$, and let

$$
\begin{aligned}
\beta_{i} & =\frac{q_{m} c_{i i} q_{1 m} r(x, t)(n-1)}{n}, \\
\alpha_{i j} & =\frac{\beta_{i} c_{i j}}{M_{i}}, \quad(i, j=\overline{1, n} ; i \neq j), \\
M_{i} & =\sum_{j=1, n ; j \neq i} c_{i j}, \quad(i=\overline{1, n}), \\
\alpha_{i(n+1)} & =\frac{q_{m} q_{1 m} r(x, t) c_{i i}}{n}, \quad(i=\overline{1, n}) .
\end{aligned}
$$

Now we will define the density function of transition matrix $P(x, t ; y, \tau)$ :

$$
\begin{aligned}
& P(x, t ; y, \tau) \\
& =\left[\begin{array}{cccc}
p_{11}(x, t ; y, \tau) & p_{12}(x, t ; y, \tau) & \ldots & p_{1(n+1)}(x, t ; y, \tau) \\
p_{21}(x, t ; y, \tau) & p_{22}(x, t ; y, \tau) & \ldots & p_{2(n+1)}(x, t ; y, \tau) \\
\ldots & \ldots & \ldots & \ldots \\
p_{n 1}(x, t ; y, \tau) & p_{n 2}(x, t ; y, \tau) & \ldots & p_{n(n+1)}(x, t ; y, \tau) \\
0 & 0 & \ldots & 1
\end{array}\right],
\end{aligned}
$$

where

$$
\begin{aligned}
& p_{i j}(x, t ; y, \tau)=p_{2}^{(i)}(x, t ; y, \tau), \\
& (i=\overline{1, n} ; j=\overline{1, n+1} ; i \neq j), \\
& p_{i i}(x, t ; y, \tau) \\
& =\frac{\left(1-q_{m}\right) p_{0}^{(i)}(x, t ; y, \tau)+q_{m}\left(1-r q_{1 m} c_{i i}\right) p_{1}^{(i)}(x, t ; y, \tau)}{1-r(x, t) q_{1 m} c_{i i} q_{m}} .
\end{aligned}
$$

Then we will fix the initial point $\left(x_{0}, t_{0}\right)=(x, t)$ and the number of equations $i_{0} \in\{1, \ldots, n\}$. Let an initial moment at the point $\left(x_{0}, t_{0}\right)=(x, t)$; we will have one particle. For one step a particle $i_{k} \rightarrow i_{k+1}$ moves from its position according to the transition matrix $A\left(x_{k}, t_{k}\right)$ and moves with probability $\alpha_{i_{k}, i_{k+1}}\left(x_{k}, t_{k}\right)$ from the point $\left(x_{k}, t_{k}\right)$ to the point $\left(x_{k+1}, t_{k+1}\right)$. The next $\left(x_{k+1}, t_{k+1}\right)$ point will be simulated using the density function $p_{i_{k} i_{k+1}}\left(x_{k}, t_{k} ; y, \tau\right)$.

The probability of breaking of trajectory in the point $\left(x_{n}, t_{n}\right)$ is

$$
g\left(x_{n}, t_{n}\right)= \begin{cases}1, & \left(x_{n}, t_{n}\right) \in \Omega ; \\ \alpha_{i_{n}, n+1}\left(x_{n-1}, t_{n-1}\right), & \left(x_{n}, t_{n}\right) \in \Omega .\end{cases}
$$

The next coordinate of the particle will be defined in the following way.

(1) If the density function of the point $\left(x_{n+1}, t_{n+1}\right)$ equals $p_{0}^{(i)}\left(x_{n}, t_{n} ; y, \tau\right)$ in the fixed point $\left(x_{n}, t_{n}\right)$ then

$$
\begin{aligned}
& x_{n+1}=x_{n}+2\left(r\left(x_{n}, t_{n}\right) \xi_{n} a_{i}\right)^{1 / 2} \exp \left(-\frac{\xi_{n}}{m}\right) \omega_{n}, \\
& t_{n+1}=t_{n}-r\left(x_{n}, t_{n}\right) \exp \left(-\frac{2 \xi_{n}}{m}\right),
\end{aligned}
$$

where $\left\{\xi_{n}\right\}_{n=0}^{\infty},\left\{\omega_{n}\right\}_{n=0}^{\infty}$ the sequence of independent random variables with the density function $q_{1}(\rho)$ and independent isotropic vectors. The value $r\left(x_{n}, t_{n}\right)$ will be defined as (50).

(2) If the density function of the point $\left(x_{n+1}, t_{n+1}\right)$ is equal to $p_{1}^{(i)}\left(x_{n}, t_{n} ; y, \tau\right)$ at a fixed $\left(x_{n}, t_{n}\right)$ then

$$
\begin{aligned}
x_{n+1}=x_{n}+2( & \frac{m}{m+2} r\left(x_{n}, t_{n}\right) \\
& \left.\times \xi_{n}^{\prime}\left(v_{n}^{\prime}\right)^{2 / m} a_{i} \exp \left(-\frac{2 \xi_{n}^{\prime}}{m+2}\right)\right)^{1 / 2} \omega_{n}, \\
t_{n+1}= & t_{n}-r\left(x_{n}, t_{n}\right)\left(v_{n}^{\prime}\right)^{2 / m} \exp \left(-\frac{2 \xi_{n}^{\prime}}{m+2}\right),
\end{aligned}
$$

where $\left\{\xi_{n}^{\prime}\right\}_{n=0}^{\infty}, \quad\left\{v_{n}^{\prime}\right\}_{n=0}^{\infty}$ is a sequence of independent random variables, which will be obtained from the algorithm below (Algorithm 4) (Neumann acceptance rejection method).

Algorithm 4. (a) We firstly simulate $\xi$, Gamma distributed random variable with the parameters $(m / 2)$, secondly simulate $\gamma$, uniformly distributed random variable on $(0,1)$, and 
thirdly simulate $v$, Beta distributed random variable with the parameters $(2,2 / m)$.

(b) If $\gamma>1-c_{i i} r\left(1-v^{2 / m} \exp (-2 \xi /(m+2))\right)$ then we will go to (a) and so on; otherwise $v^{\prime}=v, \xi^{\prime}=\xi$.

(3) If the density function at the point $\left(x_{n+1}, t_{n+1}\right)$ equals $p_{2}^{(i)}\left(x_{n}, t_{n} ; y, \tau\right)$ under fixed point $\left(x_{n}, t_{n}\right)$, then

$$
\begin{aligned}
& x_{n+1}=x_{n}+2( \frac{m}{m+2} r\left(x_{n}, t_{n}\right) \xi_{n}\left(v_{n}\right)^{2 / m} \\
&\left.\times a_{i} \exp \left(-\frac{2 \xi_{n}}{m+2}\right)\right)^{1 / 2} \omega_{n}, \\
& t_{n+1}=t_{n}-r\left(x_{n}, t_{n}\right)\left(v_{n}\right)^{2 / m} \exp \left(-\frac{2 \xi_{n}}{m+2}\right),
\end{aligned}
$$

where $\left\{\xi_{n}\right\}_{n=0}^{\infty},\left\{v_{n}\right\}_{n=0}^{\infty},\left\{\omega_{n}\right\}_{n=0}^{\infty}$ is sequence of independent Gamma distributed random variables with parameters $(2 / m)$, Beta distributed random variables with parameters $(2,2 / m)$, and independent isotropic vectors, respectively.

If at the moment $n$ was held break, then we will put $\left(x_{n+k}, t_{n+k}\right)=\left(x_{n}, t_{n}\right), k=0,1,2, \ldots$ obviously the sequence of coordinate of the particle forms Markov chains. The random process which was described above was considered in Ermakov et al. [23] for the solution of initial BVP for the heat equation and adapted in Kurbanmuradov [22] for the heat equation with variable coefficients.

Now we prove the auxiliary Lemma 5.

Lemma 5. With the probability one Markov chain $\left\{x_{n}, t_{n}\right\}_{n=0}^{\infty}$ converges when $n \rightarrow \infty$ to the random point of boundary $\left(x_{\infty}, t_{\infty}\right) \in \partial \Omega$, or it is absorbed inside of the domain.

Proof. Since $\left\{t_{n}\right\}$ is decreasing sequence and $t_{n} \geq 0$, it has a limit $t_{\infty}=\lim _{n \rightarrow \infty} t_{n}$. Let $\mathfrak{R}_{n}-\sigma$ be algebra, which was generated by random variables

$$
\begin{aligned}
& \left\{\omega_{k}\right\}_{k=0}^{n-1}, \quad\left\{v_{k}\right\}_{k=0}^{n-1}, \\
& \left\{\xi_{k}\right\}_{k=0}^{n-1}, \quad\left\{v_{k}^{\prime}\right\}_{k=0}^{n-1}, \quad\left\{\xi_{k}^{\prime}\right\}_{k=0}^{n-1} .
\end{aligned}
$$

From the definition $\mathfrak{R}_{n}$ and (57)-(59) it follows that $x_{n}$ is measurable relatively $\mathfrak{R}_{n}$. It is obvious that the coordinates of vector process formed limited martingale relatively $\left\{\mathfrak{R}_{m}\right\}_{m=1}^{\infty}$ :

$$
\begin{aligned}
& E\left(\frac{x_{n+1}}{\mathfrak{R}_{n}}\right) \\
& =E\left\{\left(1-q_{m}\right)\right.
\end{aligned}
$$

$$
\begin{aligned}
& \times\left[x_{n}+2\left(r\left(x_{n}, t_{n}\right) \xi_{n} a_{i_{n}}\right)^{1 / 2} \exp \left(-\frac{\xi_{n}}{m}\right) \omega_{n}\right] \\
& +q_{m}\left(1-q_{1 m} r\left(x_{n}, t_{n}\right) c_{i_{n} i_{n}}\right) \\
& \times\left[x_{n}+2\left(\frac{m}{m+2} r\left(x_{n}, t_{n}\right) a_{i_{n}} \xi_{n}^{\prime} v_{n}^{\prime 2 / m}\right)^{1 / 2}\right. \\
& \left.\times \exp \left(-\frac{\xi_{n}^{\prime}}{m+2}\right) \omega_{n}\right] \\
& +c_{i_{n} i_{n}} r\left(x_{n}, t_{n}\right) q_{m} q_{1 m} \frac{n-1}{n} \\
& \times\left[x_{n}+2\left(\frac{m}{m+2} r\left(x_{n}, t_{n}\right) a_{i_{n}} \varsigma_{n} v_{n}^{2 / m}\right)^{1 / 2}\right. \\
& \left.\times \exp \left(-\frac{\varsigma}{m+2}\right) \omega_{n}\right] \\
& \left.+\frac{c_{i_{n} i_{n}} r\left(x_{n}, t_{n}\right) q_{m} q_{1 m}}{n} \frac{x_{n}}{\mathfrak{R}_{n}}\right\} \\
& =\left(1-q_{m}\right)\left[x_{n}+2\left(r\left(x_{n}, t_{n}\right) a_{i_{n}}\right)^{1 / 2}\right. \\
& \left.\times E\left(\xi_{n}^{1 / 2} \exp \left(-\frac{\xi_{n}}{m}\right) \omega_{n}\right)\right] \\
& +q_{m}\left(1-q_{1 m} c_{i_{n} i_{n}} r\left(x_{n}, t_{n}\right)\right) \\
& \times\left[x_{n}+2\left(\frac{m}{m+2} r\left(x_{n}, t_{n}\right) a_{i_{n}}\right)^{1 / 2}\right. \\
& \left.\times E\left(\left(\xi_{n}^{\prime} v_{n}^{\prime 2 / m}\right) \exp \left(-\frac{\xi_{n}}{m+2}\right) \omega_{n}\right)\right] \\
& +c_{i_{n} i_{n}} r\left(x_{n}, t_{n}\right) q_{m} q_{1 m} \frac{n-1}{n} \\
& \times\left[x_{n}+2\left(\frac{m}{m+2} r\left(x_{n}, t_{n}\right) a_{i_{n}}\right)^{1 / 2}\right. \\
& \left.\times E\left(\left(\xi_{n}^{\prime} v_{n}^{\prime 2 / m}\right) \exp \left(-\frac{\xi_{n}}{m+2}\right) \omega_{n}\right)\right] \\
& +c_{i_{n} i_{n}} r\left(x_{n}, t_{n}\right) q_{m} q_{1 m} \frac{n-1}{n} \\
& \times\left[x_{n}+2\left(\frac{m}{m+2} r\left(x_{n}, t_{n}\right) a_{i_{n}}\right)^{1 / 2}\right. \\
& \left.\times E\left(\left(\varsigma_{n} v_{n}^{2 / m}\right)^{1 / 2} \exp \left(-\frac{\varsigma_{n}}{m+2}\right) \omega_{n}\right)\right] \\
& +\frac{c_{i_{n} i_{n}} r\left(x_{n}, t_{n}\right) q_{m} q_{1 m}}{n} x_{n} \\
& =\left(1-q_{m}\right) x_{n}+q_{m}\left(1-q_{1 m} c_{i_{n} i_{n}} r\left(x_{n}, t_{n}\right)\right) x_{n} \\
& +c_{i_{n} i_{n}} r\left(x_{n}, t_{n}\right) q_{m} q_{1 m} x_{n}=x_{n},
\end{aligned}
$$


where $\left\{x_{n}\right\}$ are limited martingale; it converges with the probability one Shiryaev [24].

Let $\left(x_{\infty}, t_{\infty}\right)=\lim _{n \rightarrow \infty}\left(x_{n}, t_{n}\right)$ be the limit vector. We show that $\left(x_{\infty}, t_{\infty}\right) \in \partial \Omega$. If $t_{\infty}=0$ then the process is broken inside the domain. Let $t_{\infty}>0$. As far as the process converges, according to the formulas (50)-(58) we have

$$
\begin{aligned}
& E_{\left(x_{0}, t_{0}\right)}\left|x_{n+1}-x_{n}\right| \longrightarrow 0, \\
& E_{\left(x_{0}, t_{0}\right)}\left|x_{n+1}-x_{n}\right|=E_{\left(x_{0}, t_{0}\right)}\left\{\sqrt{r\left(x_{n}, t_{n}\right)} h\left(r\left(x_{n}, t_{n}\right)\right)\right\},
\end{aligned}
$$

where $h(r)$ is strictly positive. Applying Lebesque Theorem (about the limited convergence) we get

$$
E_{\left(x_{0}, t_{0}\right)}\left(r\left(x_{\infty}, t_{\infty}\right)\right)^{1 / 2}=0 .
$$

It means $r\left(x_{\infty}, t_{\infty}\right)=0$. Then from the definition of $r(x, t)$ and using the formulae (49) we obtain

$$
R_{1}\left(x_{\infty}\right)=0, \quad\left(x_{\infty}, t_{\infty}\right) \in \partial \Omega
$$

Lemma is proven.

\section{Construction Unbiased and $\varepsilon$-Biased Estimators}

Let $\left(x_{k}, t_{k}\right)_{k=0}^{\infty}$ be the trajectories of random process which was described above. We will define on it the sequence of the random variables $\left\{\eta_{n}\left(i_{0}\right)\right\}_{n=0}^{\infty}$. Let

$$
\Theta_{0}=1, \quad \Theta_{n}=\Theta_{n-1} \times V_{i_{n-1} i_{n}}\left(x_{n-1}, t_{n-1} ; x_{n}, t_{n}\right),
$$

where $V_{i j}\left(x_{n-1}, t_{n-1} ; x_{n}, t_{n}\right)$ is defined as follows:

$$
\begin{aligned}
& V_{i j}\left(x_{n-1}, t_{n-1} ; x_{n}, t_{n}\right) \\
& =\frac{n M_{i}}{(n-1) c_{i i} q_{1 m}}\left(1-v_{n}^{2 / m} \exp \left(-\frac{2 \varsigma_{n}}{m+2}\right)\right), \\
& \qquad(i, j=\overline{1, n} ; i \neq j), \\
& V_{i i}\left(x_{n-1}, t_{n-1} ; x_{n}, t_{n}\right)=1 ; \quad(i=\overline{1, n}) ; \\
& V_{i(n+1)}\left(x_{n-1}, t_{n-1} ; x_{n}, t_{n}\right) \\
& \quad=\frac{n}{c_{i i} q_{1 m}}\left(1-v_{n}^{2 / m} \exp \left(-\frac{2 \varsigma_{n}}{m+2}\right)\right) ; \quad(i=\overline{1, n}) .
\end{aligned}
$$

Here $\left\{s_{n}\right\}_{n=0}^{\infty},\left\{v_{n}\right\}_{n=0}^{\infty}$ are the sequences of independent Gamma function with the parameters $(\mathrm{m} / 2)$ and Beta function with parameters $(2,2 / m)$ distributed random variables, respectively. We will define the sequence

$$
\begin{aligned}
\eta_{n}\left(i_{0}\right) & =\Theta_{n} \times F\left(x_{n}, t_{n}\right) \\
& =\Theta_{n} \begin{cases}u_{j}\left(x_{n}, t_{n}\right), & i_{n}=j, \quad j \neq n+1, \\
f_{i_{n-1}}\left(x_{n}, t_{n}\right), & i_{n}=n+1 .\end{cases}
\end{aligned}
$$

If at the moment $n$ happen break $n$, we will put

$$
\begin{aligned}
\eta_{n+k}\left(i_{0}\right) & =\eta_{n}\left(i_{0}\right), \\
\left(x_{n+k}, t_{n+k}\right) & =\left(x_{n}, t_{n}\right), \\
k & =1,2, \ldots,
\end{aligned}
$$

where algebra $\mathfrak{R}_{n}-\sigma$ generated until the moment $n$.

Theorem 6. Let the sequence be form martingale $\left\{\eta_{n}\left(i_{0}\right)\right\}_{n=1}^{\infty}$ with $\mathfrak{R}_{n}$, respectively. If

$$
\begin{gathered}
\sum_{j=1, \ldots, n ; j \neq i} c_{i j}<\frac{(n-1) c_{i i} q_{1 m}}{n}, \quad(i=\overline{1, n}), \\
\max _{(x, t) \in \Omega}\left|f_{i}(x, t)\right| \leq c_{0}, \quad\left(c_{0}=\text { const }, i=\overline{1, n}\right) .
\end{gathered}
$$

Then the sequence will be $\left\{\eta_{n}\left(i_{0}\right)\right\}$ uniformly integrable martingale.

Proof. From the definition $\eta_{n}\left(i_{0}\right)$ the $\mathfrak{R}_{n}$ is measurable. In this case

$$
\begin{aligned}
& E\left(\eta_{n+1} \frac{\left(i_{0}\right)}{R_{n}}\right) \\
& =E\left(\Theta_{n+1} \times \frac{F\left(x_{n+1}, t_{n+1}\right)}{\mathfrak{R}_{n}}\right) \\
& =E\left(\Theta_{n} \times V_{i_{n} i_{n+1}}\left(x_{n}, t_{n} ; x_{n+1}, t_{n+1}\right) \times \frac{F\left(x_{n+1}, t_{n+1}\right)}{\mathfrak{R}_{n}}\right) \\
& =\Theta_{n} E\left(V_{i_{n} i_{n+1}}\left(x_{n}, t_{n} ; x_{n+1}, t_{n+1}\right) \times F\left(x_{n+1}, t_{n+1}\right)\right) \\
& =\Theta_{n}\left[\sum_{j=1, n} \alpha_{i_{n} j} \iint \frac{Q_{r}^{\left(i_{n}\right)}\left(x_{n}, t_{n}\right)}{p_{i_{n} j}}\left(x_{n}, t_{n} ; y, \tau\right) u_{j}(y, \tau) d y d \tau\right. \\
& \left.\quad+\alpha_{i_{n} n+1} \iint \frac{Q_{r}^{\left(i_{n}\right)}\left(x_{n}, t_{n}\right)}{p_{i_{n} n+1}}\left(x_{n}, t_{n} ; y, \tau\right) f_{i_{n}}(y, \tau) d y d \tau\right] \\
& =\eta_{n}\left(i_{0}\right) .
\end{aligned}
$$

As far as the sequence is martingale $\left\{\eta_{n}\left(i_{0}\right)\right\}$. We can show the uniformly integrability of $\eta_{n}\left(i_{0}\right)$. To do that it is enough to show $\left|\eta_{n}\left(i_{0}\right)\right|<\infty$.

Since

$$
u_{i}(x, t) \in C(\bar{D} \times[0, T]) \cap C^{2,1}(\bar{D} \times[0, T]),
$$

and $\Omega$ is bounded domain $\left|u_{i}(x, t)\right| \leq$ const, for any $(x, t) \epsilon$ $\Omega$. From the condition of theorem $\left|\Theta_{n}\right| \leq 1$, it is followed $\left|\eta_{n}\left(i_{0}\right)\right| \leq$ const. It means $\left\{\eta_{n}\left(i_{0}\right)\right\}$ is uniformly integrable. The theorem is proved.

Now we will construct computable (realizable) estimator $\eta_{n}\left(i_{0}\right)$. We will take $\varepsilon$-neighborhoods of the domain $(\partial \Omega)_{\varepsilon}=$ $\{D \times[0, \varepsilon]\} \cup\left\{(\partial D)_{\varepsilon} \times[0, T]\right\}$. 
Let $N_{1}$ be a breaking moment of process inside of domain. $N_{\varepsilon}$ is the first passage moment $(\partial \Omega)_{\varepsilon} . N=\min \left\{N_{1}, N_{\varepsilon}\right\}$, stopping moment of process $\left\{\left(x_{n}, t_{n}\right)\right\}$. In this case the probability of absorbing will be

$$
g\left(x_{n}, t_{n}\right)= \begin{cases}1, & \left(x_{n}, t_{n}\right) \in(\partial \Omega)_{\varepsilon} \\ \alpha_{i_{n},(n+1)}\left(x_{n-1}, t_{n-1}\right), & \left(x_{n}, t_{n}\right) \in \bar{\Omega} \backslash(\partial \Omega)_{\varepsilon} .\end{cases}
$$

From Lemma 5 it follows that $N<\infty$. It could be proved that the mathematical expectation of the stopping time $\left\{\left(x_{n}, t_{n}\right)\right\}$ of Markov process is finite.

Theorem 7. Let the condition of Theorem 6 be satisfied; then the estimator $\eta_{n}\left(i_{0}\right)$ will be unbiased estimator with finite variance, where $u_{i_{0}}(x, t)$ is $i_{0}$ th component of solution vector $u(x, t)$.

Proof. Since $\eta_{n}\left(i_{0}\right)$ is uniformly integrable martingale and $N$ is Markov moment, according to the theorem in $([24,25])$ for the martingale $\left\{\eta_{n}\left(i_{0}\right)\right\}$ we obtain

$$
E_{\left(x_{0}, t_{0}\right)} \eta_{N}\left(i_{0}\right)=E_{\left(x_{0}, t_{0}\right)} \eta_{1}\left(i_{0}\right) \text {. }
$$

From the definition $\eta_{n}\left(i_{0}\right)$ holds $E_{\left(x_{0}, t_{0}\right)} \eta_{1}\left(i_{0}\right)=u_{i_{0}}(x, t)$. From condition of Theorem $6 E\left(\eta_{N}\right)^{2}\left(i_{0}\right)<\infty$ is valid accordingly the variance is finite. The theorem is proved.

Further, from $\eta_{N}\left(i_{0}\right)$ we could construct biased but computable (realizable) estimator $\eta_{N}^{*}\left(i_{0}\right)$. Let, for $x \in \partial D$, $t \in[0, T]$ and $\psi_{i}(x, t)=y_{i}(x, t)$ for $x \in D, \psi_{i}(x, 0)=$ $y_{0 i}(x),\left(x^{*}, t^{*}\right)$ closed to the point $(x, t)$ of boundary $\partial \Omega . \eta_{N}^{*}$ will be obtained with changing

$$
u_{i}\left(x_{N}, t_{N}\right) \text { in } \eta_{N}\left(i_{0}\right) \text { to } \psi_{i}\left(x_{N}^{*}, t_{N}^{*}\right) .
$$

Let us evaluate bias $\eta_{N}^{*}\left(i_{0}\right)$. It is clear that

$$
\left|E_{(x, t)} \eta_{N}\left(i_{0}\right)-u_{i_{0}}(x, t)\right| \leq E_{(x, t)}\left|\eta_{N}^{*}\left(i_{0}\right)-\eta_{N}\left(i_{0}\right)\right| .
$$

If $N=N_{1}$, in this case the process is broken when do not reach the boundary $(\partial \Omega)_{\varepsilon}$ and $\eta_{N}^{*}\left(i_{0}\right)=\eta_{N}\left(i_{0}\right)$. If $N=N_{\varepsilon}$ then $\left(x_{N}, t_{N}\right) \in(\partial \Omega)_{\varepsilon}$.

Let $A_{i}(\varepsilon)$ be a module of continuity function $u_{i}(x, t)$. In this case it is true:

$$
\left|\eta_{N}^{*}\left(i_{0}\right)-\eta_{N}\left(i_{0}\right)\right| \leq\left|\Theta_{N}\right| A(\varepsilon),
$$

where $A(\varepsilon)=\max _{i} A_{i}(\varepsilon)$, since $\left|\Theta_{N}\right| \leq 1$ then $E_{(x, t)} \mid \eta_{N}^{*}\left(i_{0}\right)-$ $u_{i_{0}}(x, t) \mid \leq A(\varepsilon)$. Finiteness of variance followed from $E \eta_{N}^{2}\left(i_{0}\right)<\infty$. The proposed algorithm we could generalize for the case with variable coefficients $c_{i j}=c_{i j}(x, t)$ and one could get the same results.

\section{Computational Example}

Let $D \in R^{3}$ be bounded domains, $\Omega=D \times[0, T]$. We will consider for some mode linitial boundary value problem

$$
\begin{aligned}
& \frac{\partial u_{i}(x, t)}{\partial t}-a_{i} \Delta u_{i}(x, t)+c_{i i} u_{i}(x, t) \\
& -\sum_{j=1,4 ; j \neq i} c_{i j} u_{j}(x, t)=f_{i}(x, t), \quad(i=\overline{1,4})
\end{aligned}
$$

for $(x, t) \in \Omega$ with the initial boundary conditions

$$
\begin{aligned}
& u_{i}(x, t)=y_{i}(x, t), \quad x \in \partial D, t \in[0, T], i=\overline{1,4}, \\
& u_{i}(x, 0)=y_{0 i}(x), \quad x \in D, i=\overline{1,4} .
\end{aligned}
$$

As domain is chosen as the simple ball, $D=\left\{\left(x_{1}, x_{2}, x_{3}\right)\right.$ : $\left.x_{1}^{2}+x_{2}^{2}+x_{3}^{2} \leq R^{2}\right\}$.

The coefficients

$$
\begin{aligned}
\left(\begin{array}{l}
a_{1} \\
a_{2} \\
a_{3} \\
a_{4}
\end{array}\right) & =\left(\begin{array}{l}
0.5 \\
0.7 \\
0.1 \\
1.0
\end{array}\right), \\
\left\{c_{i j}\right\}_{i, j=1, \ldots, 4} & =\left[\begin{array}{cccc}
2 & 0.4 & 0.5 & 0.2 \\
0.7 & 3 & 0.4 & 0.6 \\
0.3 & 0.1 & 1 & 0.1 \\
0.2 & 0.3 & 0.3 & 1.5
\end{array}\right]
\end{aligned}
$$

The initial and boundary conditions

$$
\begin{aligned}
& y_{01}(x)=x_{1}^{2}+x_{2}^{2}+x_{3}^{2} ; \\
& y_{1}(x, t)=R^{2} \exp (t) ; \\
& y_{02}(x)=\left(x_{1} x_{2} x_{3}\right)^{2} ; \\
& y_{2}(x, t)=\exp (t)\left(x_{2} x_{3}\right)^{2}\left(R^{2}-x_{2}^{2}-x_{3}^{2}\right) ; \\
& y_{03}(x)=\exp \left(x_{1}+x_{2}+x_{3}\right) ; \\
& y_{3}(x, t)=\exp \left(t+x_{1}+x_{2}+x_{3}\right) ; \\
& y_{04}(x)=1 ; \quad y_{4}(x, t)=\exp \left(t x_{1} x_{2} x_{3}\right) .
\end{aligned}
$$

Left hand sides

$$
\begin{aligned}
f_{1}(x, t)= & \exp (t)\left[4\left(x_{1}^{2}+x_{2}^{2}+x_{3}^{2}\right)-0.3\left(x_{1} x_{2} x_{3}\right)^{2}-3\right] \\
& -0.2\left(x_{1}+x_{2}+x_{3}+t\right)-0.5 \exp \left(x_{1} x_{2} x_{3} t\right) \\
f_{2}(x, t)= & \exp (t)\left[3.5\left(x_{1} x_{2} x_{3}\right)^{2}-1.4\left(x_{1} x_{2}\right)^{2}\right. \\
& \left.+\left(x_{2} x_{3}\right)^{2}+\left(x_{1} x_{3}\right)^{2}-0.4\left(x_{1}^{2}+x_{2}^{2}+x_{3}^{2}\right)\right] \\
& -0.3\left(x_{1}+x_{2}+x_{3}+t\right)-0.2 \exp \left(x_{1} x_{2} x_{3} t\right)
\end{aligned}
$$


TABLE 1: The results of computational experiments.

\begin{tabular}{lccccccc}
\hline$\left(x_{0}, t_{0}\right)$ & $i_{0}$ & $N_{t}$ & $\varepsilon$ & $U_{e}$ & MC & $3 \sigma$ & 0.1925 \\
$(0.1 ;-0.8 ; 0.4 ; 1.2)$ & 1 & 5000 & 0.005 & 2.689294 & 2.881862 & 0.2821 & 0.224699 \\
$(0.1 ;-0.8 ; 0.4 ; 1.2)$ & 2 & 5000 & 0.005 & 0.003399 & 0.018739 & 0.21815 & 0.02423 \\
$(0.1 ;-0.8 ; 0.4 ; 1.2)$ & 3 & 5000 & 0.005 & 2.459603 & 2.483835 & 0.95895 & 0.03377 \\
$(0.1 ;-0.8 ; 0.4 ; 1.2)$ & 4 & 5000 & 0.005 & 0.962327 & 1.554352 & 0.32319 & 0.1598 \\
$(-0.5 ; 0.25 ;-0.4 ; 1.3)$ & 1 & 5000 & 0.005 & 1.733942 & 0.17939 \\
$(-0.5 ; 0.25 ;-0.4 ; 1.3)$ & 2 & 5000 & 0.005 & 0.009173 & 0.009841 & 0.215628 & 0.000668 \\
$(-0.5 ; 0.25 ;-0.4 ; 1.3)$ & 3 & 5000 & 0.005 & 1.91554 & 1.980669 & 0.18057 & 0.064528 \\
$(-0.5 ; 0.25 ;-0.4 ; 1.3)$ & 4 & 5000 & 0.005 & 1.067159 & 1.084443 & 0.162095 & 0.01728 \\
\hline
\end{tabular}

$\left(x_{0} ; t_{0}\right)$ is the point which solved BVP; $N_{t}$ is the quantity of samples (trajectories); $\varepsilon$ is neighborhood area; $i_{0}$ is the number of equation; $U_{e}$ is exact solution at the point $\left(x_{0} ; t_{0}\right)$. MC is Monte Carlo solutions; $3 \sigma$ is confidence interval; err is the difference between exact ant MC solutions err $=\mid u_{i_{0}}\left(x_{0} ; t_{0}\right)-$ MS $\mid$.

$$
\begin{aligned}
f_{3}(x, t)= & 4.7 \exp \left(x_{1}+x_{2}+x_{3}+t\right) \\
& -\exp (t)\left[0.5\left(x_{1}^{2}+x_{2}^{2}+x_{3}^{2}\right)+0.4\left(x_{1} x_{2} x_{3}\right)^{2}\right] \\
& -0.7 \exp \left(x_{1} x_{2} x_{3} t\right) ; \\
f_{4}(x, t)= & \exp \left(x_{1} x_{2} x_{3} t\right) \\
\times & {\left[\left(x_{1} x_{2} x_{3}\right)+3.5-t^{2}\left(\left(x_{1} x_{2}\right)^{2}\right.\right.} \\
& \left.\left.\quad+\left(x_{2} x_{3}\right)^{2}+\left(x_{1} x_{3}\right)^{2}\right)\right] \\
& -\exp (t)\left[0.3\left(x_{1}^{2}+x_{2}^{2}+x_{3}^{2}\right)-0.4\left(x_{1} x_{2} x_{3}\right)^{2}\right] \\
& -0.6\left(x_{1}+x_{2}+x_{3}+t\right) .
\end{aligned}
$$

The exact solutions are known:

$$
\begin{aligned}
& u_{1}(x, t)=\exp (t)\left(x_{1}^{2}+x_{2}^{2}+x_{3}^{2}\right), \\
& u_{2}(x, t)=\exp (t)\left(x_{1} x_{2} x_{3}\right)^{2}, \\
& u_{3}(x, t)=\exp \left(x_{1}+x_{2}+x_{3}+t\right), \\
& u_{4}(x, t)=\exp \left(x_{1} x_{2} x_{3} t\right) .
\end{aligned}
$$

\section{Conclusions}

It is known that the distinguishing feature of the vector algorithm is that its "weight" appears to be a matrix weight. This matrix weight is multiplied by the kernel matrix of the system of integral equations divided by a transition density function after each transition in the Markov chain simulation. In this case the computational complexity is higher enough than simple Monte Carlo method. On the contrary to the vector algorithms we proposed a new Monte Carlo algorithm for the solution of system of integral equations. This method has the simple structure of the computation algorithm and the errors do not depend on the dimension of domain and smoothness of boundary. One can solve the problem at one point and we do not use matrix weights. Proposed algorithm applied to the solution of system of the parabolic equations. To do so we derived corresponding system of integral equations and construct a special probabilistic representation. This probabilistic representation uses for simulation the random process and construction the unbiased and $\varepsilon$-biased estimator for the solution of systems IEs.

Numerical experiments show that the computational complexity of our algorithm is reduced. In the future the proposed algorithm might be generalized for the case with variable coefficients $c_{i j}=c_{i j}(x, t)$. The results of numerical experiments are shown with the probability almost one; the approximate solution tends to the exact solution of the problem. In the given example the exact solution is known; therefore we can make sure that all the estimators really are in the confidence intervals (see Table 1).

\section{Conflict of Interests}

The authors declare that they have no conflict of interests regarding publication of this paper and they do not have direct financial relation that might lead to conflict of interests for any of the authors.

\section{Acknowledgments}

The authors would like to thank the anonymous referees, especially the second referee, for their constructive comments and suggestions.

\section{References}

[1] H. M. Srivastava and R. G. Buschman, Theory and Applications of Convolution Integral Equations, vol. 79 of Mathematics and Its Applications, Kluwer Academic, Dodrecht, The Netherlands, 1992.

[2] T. A. Burton, Volterra Integral and Differential Equations, vol. 202 of Mathematics in Science and Engineering, Elsevier, Amsterdam, The Netherlands, 2005.

[3] H. Brunner, Collocation Method for Volterra Integral and Related Functional Equations, Cambridge University Press, Cambridge, UK, 2004.

[4] L. M. Delves and J. L. Mohamed, Computational Methods for Integral Equations, Cambridge University Press, Cambridge, UK, 1985.

[5] E. Yusufoğlu, "Numerical solving initial value problem for Fredholm type linear integro-differential equation system," 
Journal of the Franklin Institute, vol. 346, no. 6, pp. 636-649, 2009.

[6] J. Pour-Mahmoud, M. Y. Rahimi-Ardabili, and S. Shahmorad, "Numerical solution of the system of Fredholm integrodifferential equations by the Tau method," Applied Mathematics and Computation, vol. 168, no. 1, pp. 465-478, 2005.

[7] A. Arikoglu and I. Ozkol, "Solutions of integral and integrodifferential equation systems by using differential transform method," Computers and Mathematics with Applications, vol. 56, no. 9, pp. 2411-2417, 2008.

[8] K. Maleknejad, M. Shahrezaee, and H. Khatami, "Numerical solution of integral equations system of the second kind by block-pulse functions," Applied Mathematics and Computation, vol. 166, no. 1, pp. 15-24, 2005.

[9] K. Maleknejad, F. Mirzaee, and S. Abbasbandy, "Solving linear integro-differential equations system by using rationalized Haar functions method," Applied Mathematics and Computation, vol. 155, no. 2, pp. 317-328, 2004.

[10] S. Yüzbaşı, N. Şahin, and M. Sezer, "Numerical solutions of systems of linear Fredholm integro-differential equations with Bessel polynomial bases," Computers and Mathematics with Applications, vol. 61, no. 10, pp. 3079-3096, 2011.

[11] A. Tahmasbi and O. S. Fard, "Numerical solution of linear Volterra integral equations system of the second kind," Applied Mathematics and Computation, vol. 201, no. 1-2, pp. 547-552, 2008.

[12] M. Rabbani, K. Maleknejad, and N. Aghazadeh, "Numerical computational solution of the Volterra integral equations system of the second kind by using an expansion method," Applied Mathematics and Computation, vol. 187, no. 2, pp. 1143-1146, 2007.

[13] P. J. van der Houwen and B. P. Sommeijer, "Euler-Chebyshev methods for integro-differential equations," Applied Numerical Mathematics, vol. 24, no. 2-3, pp. 203-218, 1997.

[14] A. G. Ramm, "Investigation of a class of systems of integral equations," Journal of Mathematical Analysis and Applications, vol. 76, no. 2, pp. 303-308, 1980.

[15] M. C. de Bonis and C. Laurita, "Numerical treatment of second kind Fredholm integral equations systems on bounded intervals," Journal of Computational and Applied Mathematics, vol. 217, no. 1, pp. 64-87, 2008.

[16] A. R. Vahidi and M. Mokhtari, "On the decomposition method for system of linear Fredholm integral equations of the second kind," Journal of Applied Mathematical Sciences, vol. 2, no. 2, pp. 57-62, 2008.

[17] G. A. Mikhailov, Optimization of Weighted Monte Carlo Methods, Springer, 1992.

[18] O. A. Ladyzhenskaya, V. A. Solonnikov, and N. N. Ural'tseva, Linear and Quasilinear Equations of Parabolic Type, American Mathematical Society, 1968.

[19] J. Lions, Optimal Control of Systems Governed by Partial Differential Equations, vol. 170 of Grundlehren der Mathematischen Wissenschaften, 1971.

[20] L. P. Kupcov, "The mean property and the maximum principle for parabolic equations of second order," Soviet Mathematics. Doklady, vol. 19, no. 5, pp. 741-747, 1978.

[21] A. Rasulov, M. Mascagni, and G. Raimova, Monte Carlo Methods for Solution Linearand Nonlinear Boundary Value Problems, Monograph, UWED Press, Tashkent, Uzbekistan, 2006.

[22] O. Kurbanmuradov, Random walks for non-stationary problems of mathematical physics, [PhD thesis], Novosibirsk, Russia, 1984, (Russian).
[23] S. M. Ermakov, V. V. Nekrutkin, and A. S. Sipin, Random Processes for Classical Equations of Mathematical Physics, Kluwer Academic Publishers, 1989.

[24] A. N. Shiryaev, Probability, Springer, New York, NY, USA, 1995.

[25] P. A. Meyer, Probability and Potentials, Blasdell Publishing, Waltham, Mass, USA, 1966. 


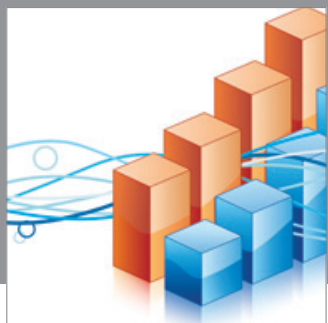

Advances in

Operations Research

mansans

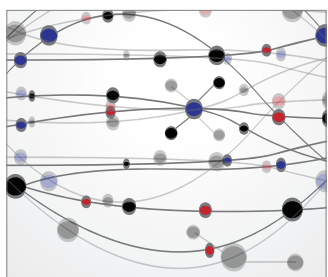

The Scientific World Journal
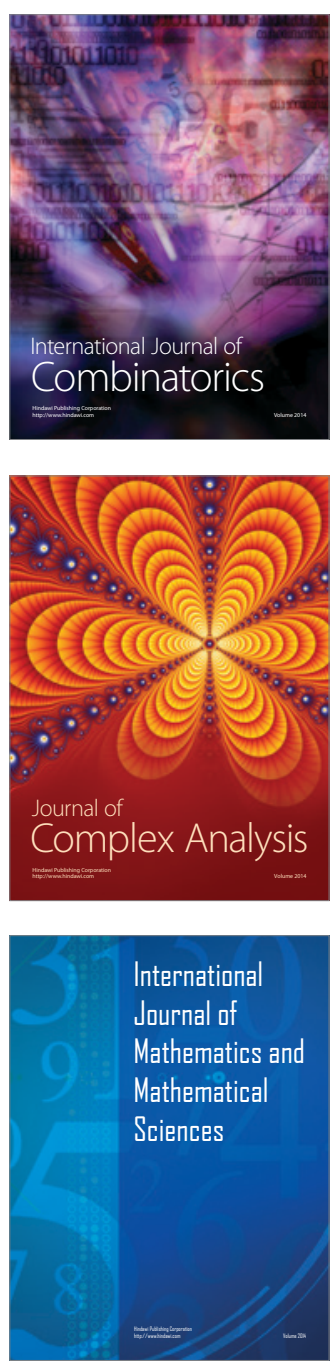
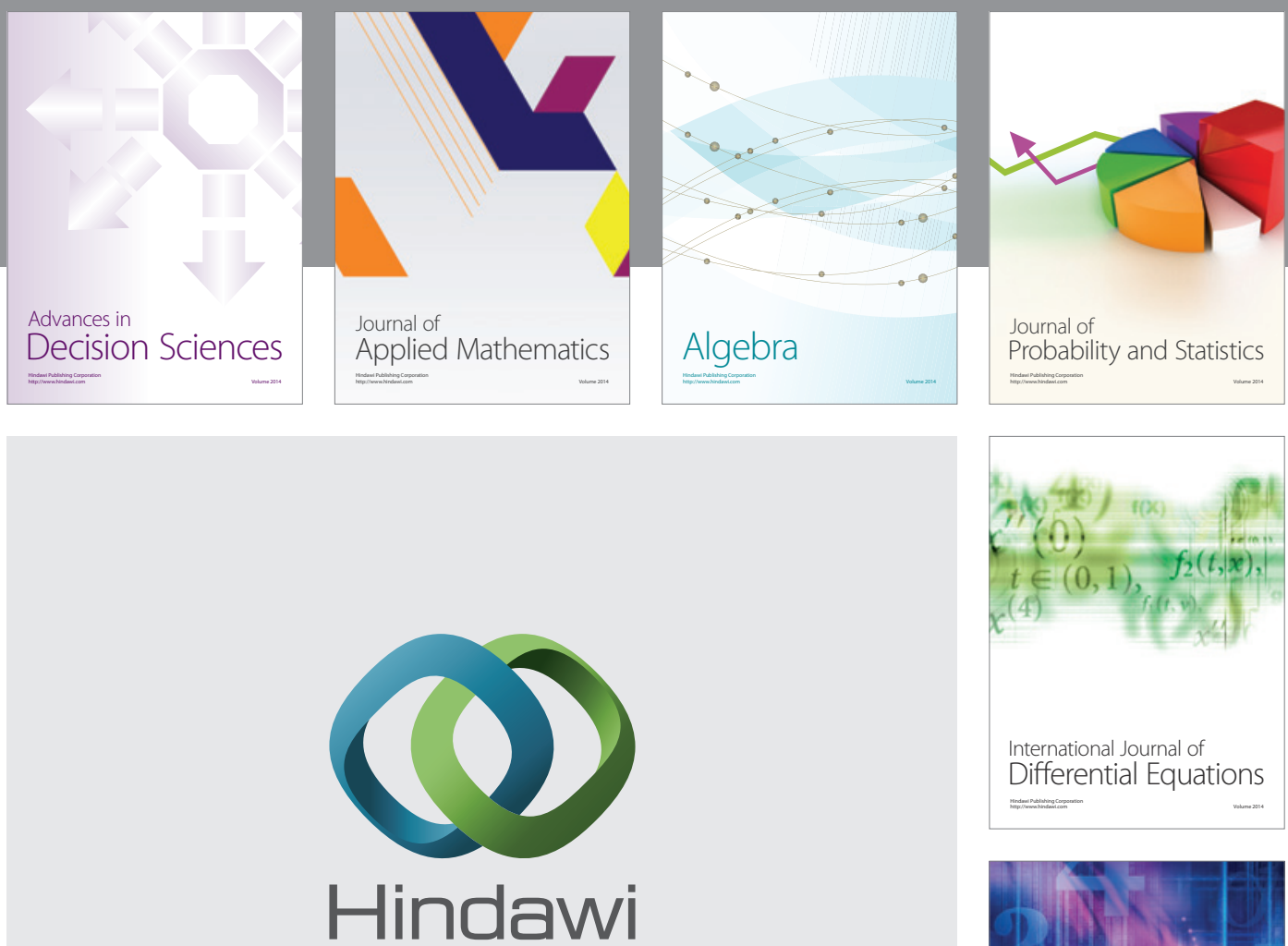

Submit your manuscripts at http://www.hindawi.com
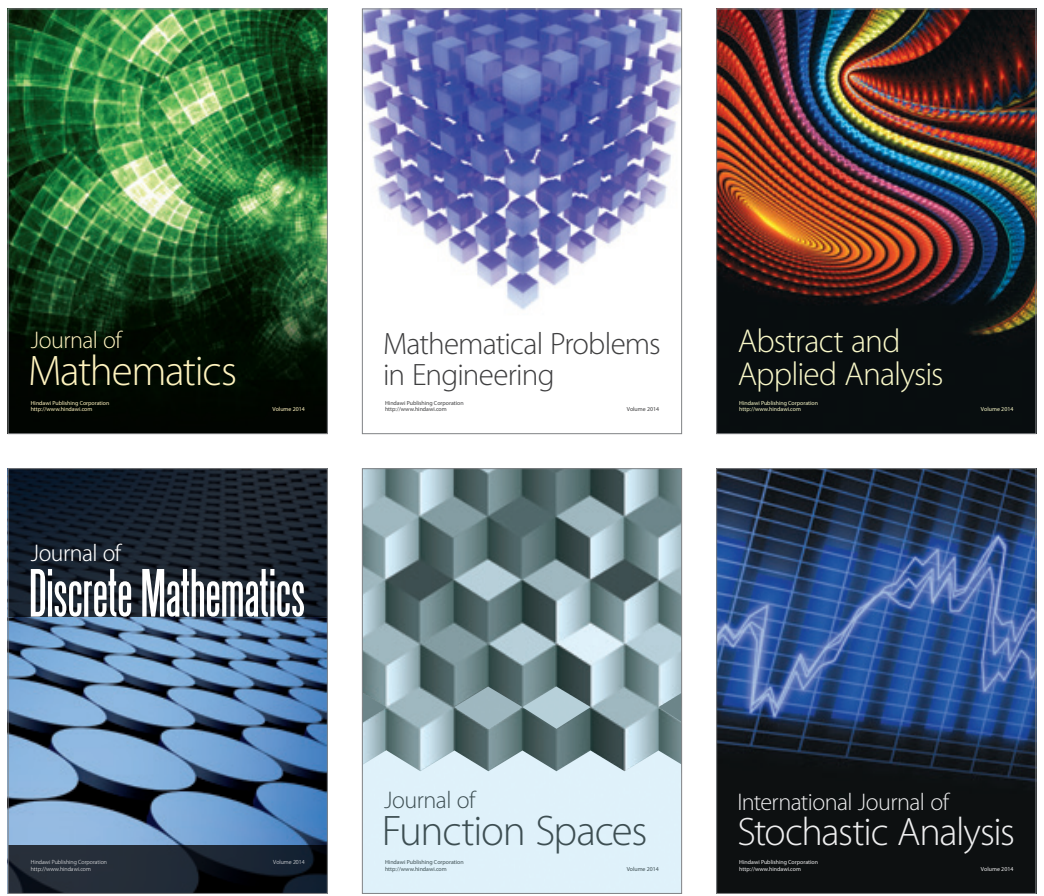

Journal of

Function Spaces

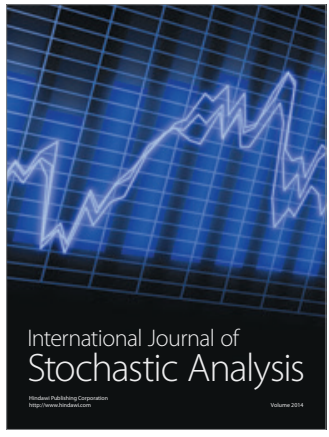

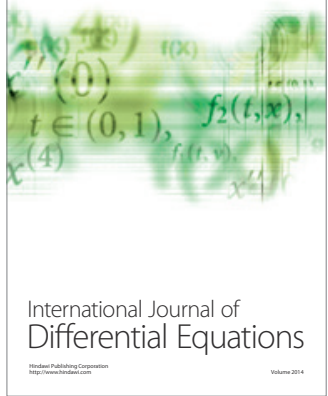
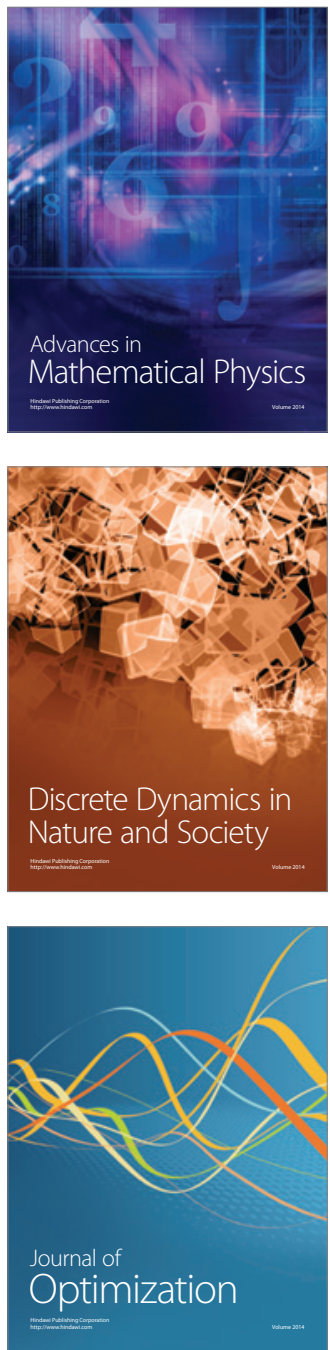